\title{
POTENCIAIS INTERAÇÕES MEDICAMENTOSAS EM PRESCRIÇÕES CONTENDO PSICOFÁRMACOS DE PACIENTES INTERNOS EM UM HOSPITAL TERCIÁRIO
}

\author{
R.E. ALVES ${ }^{1}$, F. P. B. F. CARDOSO ${ }^{2}$, V.M. SILVA ${ }^{3}$, H. L. SILVA-NETA ${ }^{4}$, R. M. S.M. \\ SERRANO ${ }^{5}$
}

\footnotetext{
${ }^{1}$ Universidade Federal da Paraíba, Centro de Ciências da Saúde, Núcleo de Saúde Coletiva

${ }^{2}$ Universidade Federal da Paraíba, Hospital Universitário Lauro Wanderley, Unidade de Farmácia Clínica

${ }^{3}$ Universidade Federal da Paraíba, Centro de Ciências da Saúde, Núcleo de Saúde Coletiva

${ }^{4}$ Universidade Federal de Pernambuco, Núcleo de Pesquisa em Inovação Terapêutica Suely Galdino, Laboratório de Imunomodulação e Novas Abordagens Terapêuticas

${ }^{5}$ Universidade Federal da Paraíba, Centro de Ciências da Saúde - Campus I, Departamento de Ciências Farmacêuticas
}

E-mail para contato: silvanetahlj@gmail.com

RESUMO - Tendo em vista a relevância das prescrições de psicofármacos em hospitais gerais, o objetivo deste trabalho foi investigar as potenciais interações entre fármacos e psicofármacos nas prescrições médicas de pacientes internos, da clínica médica A, do Hospital Universitário Lauro Wanderley (HULW). Para tanto, foram analisadas todas as prescrições contendo pelo menos um psicofármaco, referentes ao periodo de abril a maio de 2016. A detecção das possíveis interações foi realizada com a utilização da base de dados eletrônica Micromedex ${ }^{\circledR}$. Todo o estudo foi desenvolvido em parceria entre a Residência Multiprofissional em Saúde Mental/UFPB/NESC e a Farmácia Clínica do HULW. Com relação aos psicofármacos, os fármacos potencialmente interativos, ou seja, aqueles mais envolvidos em Interações Medicamentosas Potenciais (IMP), foram a sertralina com 15,7\% $(n=14)$, o clonazepam com 14,6\% $(n=13)$ e o haloperidol com 12,3\% $(n=11)$. Com relação as IMP com maior frequência, houve uma preponderância de IMP entre Clonazepam e Tramadol $(7,9 \%, n=7) e$ entre Sertralina e Dipirona 5,6\%, n=5). Ambas classificadas como IMP importantes com relação a gravidade. Apesar do elevado alto índice de prescrições relacionadas a psicofármacos, não foi observada uma excessiva associação de psicofármacos nas prescrições médicas do HULW, o que reduz a possibilidade de IMP.

Palavras-chave: Saúde Mental. Polifarmácia. Interação medicamentosa

ABSTRACT - The objective of this study was to investigate the potential interactions between drugs and psychotropic drugs in the internal medical 
prescriptions of the A medical clinic of the Lauro Wanderley University Hospital (HULW), in view of the relevance of the prescriptions of psychotropic drugs in general hospitals. To do so, all prescriptions containing at least one psychotropic drug, referring to the period from April to May 2016, were analyzed. The detection of possible interactions was performed using the Micromedex ${ }^{\circledR}$ electronic database. The entire study was developed in partnership between the Multiprofessional Residency in Mental Health / UFPB / NESC and the HULW Clinical Pharmacy. Among potentially psychoactive drugs, potentially interacting drugs, ie those most involved in Potential Drug Interactions (IMP), were sertraline with $15.7 \%(n=14)$, clonazepam with $14.6 \%(n=13)$ and haloperidol with $12.3 \%(n=11)$. With regard to IMPs more frequently, there was a preponderance of IMP between Clonazepam and Tramadol $(7.9 \%, n=7)$ and between Sertraline and Dipirone 5.6\%, $n=5$ ). Both are classified as important IMPs with respect to severity. Despite the high index of prescriptions related to psychopharmaceuticals, an excessive association of psychoactive drugs in the medical prescriptions of HULW was not observed, which reduces the possibility of IMP.

Keywords: Mental Health. Polypharmacy. Drug Interaction

\section{INTRODUÇÃO}

Os psicofármacos podem ser definidos como drogas que atuam a nível de Sistema Nervoso Central e que alteram o humor e comportamento dos indivíduos, constituindo estratégia terapêutica de escolha para os transtornos psiquiátricos (RANG, 2004). Os principais psicofármacos em uso na atualidade são: ansiolíticos e hipnóticos, antidepressivos, antipsicóticos ou neurolépticos e estabilizadores do humor. (CORDIOLI, 2000).

Para se trabalhar os transtornos psiquiátricos, o uso de medicamentos costuma ser fundamental, entretanto, se faz necessário o trabalho de uma equipe multidisciplinar com a utilização de outras abordagens, como a psicoterapia; terapia ocupacional; orientação familiar; abordagem psicossocial; grupos de auto-ajuda; no intuito de se atingir a reabilitação completa do usuário, para além da condição fisiológica com o tratamento medicamentoso (SHIRAKAWA, 2000).

Nos últimos anos a utilização de psicofármacos tem aumentado de considerável e alarmante, o que se atribui ao maior numero de diagnósticos de transtornos mentais na população, lançamento de novos medicamentos e uso dos psicofarmácos também para outros fins terapêuticos (RODRIGUES et al., 2006).

O elevado grau de uso de psicofármacos está relacionado ao poder que a medicina tem em posse das novas rotulações diagnósticas e de tratamentos para esses problemas (FERRAZA et al., 2010). Mediante essa situação, conhece-se o chamado uso abusivo de medicamento, indo contra o que é preconizado pela Organização Mundial da Saúde, quando se fala em uso racional e adequado do medicamento avaliando-se sua condição clínica (WHO, 1987).

As patologias psíquicas são tratadas com o uso simultâneo de vários medicamentos, o que está diretamente relacionada ao risco de interações medicamentosas. Essa politerapia é utilizada quando se deseja obter efeito terapêutico sinergético, acarretando assim uma maior eficácia no tratamento (CORDIOLI, 2000). 
Entretanto, as combinações medicamentosas podem resultar em interações indesejadas, desencadeando vários problemas e reações adversas.

Interação medicamentosa (IM) é uma resposta farmacológica ou clínica a administração de uma combinação de medicamentos, diferente dos efeitos dos agentes utilizados individualmente, podendo resultar em aumento ou diminuição da eficácia de um ou mais princípios ativos, ou promover o aparecimento de um novo efeito que não ocorreu com um dos fármacos isoladamente (MINISTÉRIO DA SAÚDE, 2009). A frequência de IM aumenta exponencialmente com o número de fármacos prescritos, podendo contribuir para uma hospitalização ou até mesmo levar ao óbito (BACHMANN, 2006; BECKER, 2006). Dos fatores de risco relacionados com o doente, a polimedicação surge como o mais preocupante.

Tendo em vista a relevância atual da prescrição de psicofármacos em hospitais gerais, e na perspectiva de contribuir com a compreensão dos profissionais da saúde mental sobre a ocorrência e o manejo clínico das Interações Medicamentosas Potenciais (IMP), o presente estudo objetivou avaliar o perfil das interações medicamentosas potenciais em prescrições médicas de pacientes internados eum hospital terciário.

\section{MATERIAIS E MÉTODOS}

Foi desenvolvido um estudo retrospectivo, descritivo, documental e transversal, desenvolvido em parceria com a Residência Multiprofissional em Saúde Mental/UFPB/NESC e a Farmácia Clínica do HULW. As fontes de dados utilizadas compreenderam as prescrições arquivadas no serviço de Farmácia Clínica do referido hospital no período de abril a maio de 2016. Foram analisadas todas as prescrições contendo pelo menos um psicofármaco prescrito, realizadas no período citado, sendo registrada apenas uma prescrição por prontuário. Todas as prescrições com registro de psicofármacos do período de abril a maio de 2016 contabilizaram 48 prescrições. Os dados foram coletados no período de outubro e novembro de 2016 e, colhidas informações referentes aos medicamentos prescritos e data da dispensação, além do levantamento das demais variáveis abordadas no estudo (idade e sexo do paciente). Foi realizada uma consulta à base de dados Micromedex Health Series, para a busca e classificação das IMP segundo os critérios de gravidade, documentação e mecanismo de ação. Os dados armazenados foram transferidos para o software SPSS 11.5, para a análise estatística. A pesquisa foi submetida e aprovada pelo Comitê de Ética em Pesquisa do HULW (CAAE: 58527316.0.0000.5183).

\section{RESULTADOS E DISCUSSÃO}

Foram coletadas 48 prescrições no período estudado, a partir da análise destas prescrições obteve-se uma média de 11,5 medicamentos por prescrição que ultrapassa a faixa recomendada pela Organização Mundial de Saúde (OMS), compreendida entre 1,3 a 2,2 (BECKER at al, 2006); além disso, as prescrições com cinco ou mais medicamentos corresponderam a 95,8\% das prescrições totais. Esta informação indica uma tendência a polifarmácia, ou seja, o uso concomitante de dois ou mais medicamentos pelo mesmo sujeito, o que predispõe a ocorrência de IMP.

A média de internações por mês de usuários foi mensurada a partir de dados coletados na farmácia clínica, o que resultou em 58,5 ao mês. Em dois meses totalizaria 117 usuários internados. A amostra estudada abrangeu prescrições únicas de 48 usuários, 
ou seja, para 48,02\% dos pacientes internados, foram prescritos pelo menos um psicofármaco. Indicando um alto número de prescrições relacionadas a psicofármacos. Destes, $37,5 \%$ eram homens e $62,5 \%$ eram mulheres. A média de idade destes usuários é de 54,5 anos, e os pacientes com 60 anos ou mais compreenderam $41,7 \%$ do total de usuários de psicofármacos.

Pôde-se observar que o número de mulheres com prescrições contendo um ou mais psicofármaco, no período estudado, foi maior que o número de homens, o que é corroborado por vários estudos e atribuído a maior preocupação das mulheres com a própria saúde e a maior prevalência da ansiedade e depressão (ANDRADE et al, 2006).

As alterações fisiológicas e fisiopatológicas inerentes à idade determinam uma farmacocinética mais complexa e um risco acrescido de IM. Os doentes idosos estão mais vulneráveis a este tipo de IM devido a alterações como o aumento do $\mathrm{pH}$ gástrico, diminuição da superfície de absorção, da motilidade intestinal e do débito sanguíneo (BATES et al., 2011).

Neste trabalho não foi possível observar uma associação entre idade e número de medicamentos prescritos e potencial de ocorrência de interações medicamentosas. Este fato pode ter ocorrido, por ter sido estudado a prescrição e não o usuário em si. Além disso, foram estudadas apenas uma prescrição por usuário, podendo ocorrer uma subestimação da quantidade de medicamentos prescritos. Dessa forma, os resultados, no que se refere a faixa etária dos usuários, expõe apenas uma parte do problema de interações entre medicamentos em pacientes idosos, um grupo geralmente exposto a um número maior de doenças. (Tamblyn et al, 1996).

No que diz respeito a frequência de psicofármacos prescritos por prescrição, temse que $77,1 \%$ das prescrições possuem 01 picofármaco prescrito, em $18,7 \%$ das prescrições possuem 02 psicofármacos prescritos e em $4,2 \%$ das prescrições possuem 03 psicofármacos prescritos. Desta forma, pode-se observar que houve um número menor de associação entre psicofármacos, visto que a maior parte das prescrições continham apenas 01 psicofármaco prescrito, ou seja, não existe uma excessiva associação de psicofármacos no HULW.

Tabela 01: Ocorrência da quantidade de psicofármacos por prescrição

\begin{tabular}{ccc}
\hline Psicofármaco/prescrição & N & $\mathbf{( \% )}$ \\
\hline 01 & 37 & 77,1 \\
02 & 09 & 18,7 \\
03 & 02 & 4,2 \\
\hline
\end{tabular}

Fonte: Prescrições ambulatoriais, do HULW, João Pessoa, Paraíba, abri. / mai. 2016.

No total, foram prescritos 61 psifcofármacos. Dentre estes, foram identificados 18 princípios ativos. Os mais frequentemente prescritos foram o clonazepam com 41,7\%, pertencente a classe dos benzodiazepínicos ansiolíticos, sertralina com 16,7\%, pertencente a classe dos antidepressivos Inibidores Seletivos da Recaptação da Serotonina (ISRS) e Haloperidol com 10,4\%, pertencente a classe dos antipsicóticos ou neurolépticos.

Tabela 02: Frêquencia de Psicofármacos mais prescritos

\begin{tabular}{ccc}
\hline Psicofármaco & (N) & (\%) \\
\hline Clonazepam & 20 & 41,7 \\
Sertralina & 08 & 16,7 \\
Haloperidol & 05 & 10,4 \\
\hline
\end{tabular}


Fonte: Prescrições médicas do HULW, João Pessoa, Paraíba, abri. / mai. 2016.

Foram encontradas 330 interações medicamentosas potenciais de todas as classes medicamentosas indicadas neste estudo, com uma média de 7 IMP por prescrição. De acordo com a plataforma Micromedex ${ }^{\circledR}$, as IMP de todos as classes de fármacos prescritos foram classificadas e numeradas. Interações importantes totalizaram 187, interações moderadas totalizaram 130, associações secundárias totalizaram $08 \mathrm{e}$ associações contraindicadas totalizaram 05. Das 330 interações medicamentosas potencias, 89 aproximadamente $(27 \%)$, envolvem psicofármacos.

Tabela 03: Frequência de Interações medicamentosas potenciais (IMP) de todas as classes medicamentosas, em prescrições ambulatoriais, segundo critérios de gravidade.

\begin{tabular}{ccc}
\hline Critério de Classificação & N & \% \\
\hline Importante & 187 & 56,7 \\
Moderada & 130 & 39,4 \\
Secundária & 008 & 02,4 \\
Contraindicado & 005 & 01,5 \\
\hline
\end{tabular}

Fonte: Prescrições médicas, do HULW, João Pessoa, Paraíba, abri. / mai. 2016.

Tabela 04: Frequência de Interações medicamentosas potenciais (IMP) envolvendo psicofármacos em prescrições ambulatoriais, segundo critérios de gravidade.

\begin{tabular}{ccc}
\hline Critério de Classificação & $\mathbf{N}$ & $\mathbf{\%}$ \\
\hline Importante & 65 & 73,0 \\
Moderada & 20 & 22,5 \\
Secundária & 04 & 04,5 \\
Contraindicado & 01 & 01,1 \\
\hline
\end{tabular}

Fonte: Prescrições médicas do HULW, João Pessoa, Paraíba, abri. / mai. 2016.

Nas tabelas acima, pode-se observar que tanto nas interações contendo todas as classes de fármacos prescritos como nas interações relacionadas a psicofármacos, houve uma preponderância de IMP classificadas como importantes, retificando a importância tanto da atenção dos médicos ao prescrever como do farmacêutico na área da farmácia clínica para identificar essas possíveis interações, visto que, a interação classificada como importante pode representar perigo à vida e/ou requerer atenção médica para diminuir ou evitar efeitos adversos graves, de acordo com a base MICROMEDEX.

Com relação aos psicofármacos, os fármacos potencialmente interativos, ou seja, aqueles mais envolvidos em IMP, foram a sertralina com 15,7\% $(n=14)$, o clonazepam com $14,6 \%(n=13)$ e o haloperidol com $12,3 \%(n=11)$.

Tabela05: Frequência de psicofármacos potencialmente interativos envolvidos em IMP em prescrições ambulatoriais.

\begin{tabular}{ccc}
\hline Psicofármacos Potencialmente Interativos & $(\mathbf{N})$ & $(\%)$ \\
\hline Sertralina & 14 & 15,7 \\
Clonazepam & 13 & 14,6 \\
Haloperidol & 11 & 12,3
\end{tabular}

Fonte: Prescrições médicas do HULW, João Pessoa, Paraíba, abri. / mai. 2016. 
Com relação as IMP com maior frequência, houve uma preponderância de IMP entre Clonazepam e Tramadol $(7,9 \%, n=7)$ e entre Sertralina e Dipirona 5,6\%, $n=5)$. Ambas classificadas como IMP importantes com relação a gravidade.

Tabela 06: Principais interações medicamentosas potenciais (IMP) relacionadas a psicofármacos

\begin{tabular}{ccc}
\hline Principais IMP & N & \% \\
\hline Clonazepam e Tramadol & 7 & 7,9 \\
Sertralina e Dipirona & 5 & 5,6
\end{tabular}

Fonte: Prescrições médicas do HULW, João Pessoa, Paraíba, abri. / mai. 2016.

A IMP entre Clonazepam e Tramadol está documentada de forma razoável (a documentação disponível é insatisfatória, mas as considerações farmacológicas, levam os clínicos a suspeitar da existência da interação; ou a documentação é boa para um medicamente farmacologicamente similar) na literatura científica. $\mathrm{O}$ uso concomitante de tramadol com outros depressores do SNC pode resultar em sedação profunda, depressão respiratória, coma e / ou morte. Aconselha-se reservar o uso concomitante em situações clínicas em que as opções de tratamento alternativas sejam inadequadas (US Food and Drug Administration (FDA), 2016).

A IMP entre Sertralina e Dipirona está classificada com o critério de excelência (estudos controlados estabelecem de modo claro a existência da interação) na literatura cientifica pela base de dados MICROMEDEX. Recomenda-se precaução com o uso concomitante de AINEs e antidepressivos, incluindo SSRIs devido a um risco aumentado de hemorragia intracraniana e sangramento gastrointestinal.

\section{CONCLUSÃO}

O processo de assimilação e categorização das IMP é de fundamental importância para evitar ou minimizar os seus efeitos nos usuários, destacando a farmácia clínica e a participação do farmacêutico como medida potencial para manejo clínico adequado das IMP.

O uso dos medicamentos pelo HULW reforça o alto índice de prescrições relacionadas a psicofármacos, porém não existe uma excessiva associação de psicofármacos nas prescrições médicas do HULW, diminuindo também a possibilidade de IMP.

O estudo demonstra que a classe de medicamentos ansiolíticos e antidepressivos são os mais prescritos, corroborando com o fato de que pacientes que estão hospitalizados, apresentam condições crônicas com potencial para uso de diversos medicamentos, além de elevada prevalência de transtornos psiquiátricos.

As informações discutidas neste trabalho possibilitaram uma reflexão sobre a atuação das equipes multidisciplinares no contexto hospitalar, não esgotam a temática e rementem a aprofundamentos através de outros estudos, sendo importante repensar sobre as formas de intervenções propostas no cuidado pelo HULW.

\section{REFERÊNCIAS}

ANDRADE, L. H. S.G.; VIANA, M. C.; SILVEIRA, C. M. Epidemiologia dos transtornos psiquiátricos na mulher. Rev. psiquiatr. clín. vol.33 no.2 São Paulo, 2006 
BACHMANN, KA. Interações medicamentosas: o novo padrão de interações medicamentosas e fitoterápicas. 2. ed. Barueri, SP: Manole, 2006.

BECKER ML, KALLEWAARD M, CASPERRS PW, VISSER LE, LEUFKENS HGM, STRICKER, BH. Hospitalisations and emergency department visits due to drug-drug interactions: a literature review. Pharmacoepidemiol Drug Saf. 2006 jun; 16(6): 64151.

CORDIOLI, Aristides Volpato . Psicofármacos nos transtornos mentais. Ed. Artmed, 2000 .

Ministério da Saúde (Brasil). Agência Nacional de Vigilância Sanitária (ANVISA). Resolução da Diretoria Colegiada no 47 de 08 de setembro de 2009. Diário Oficial da União, Poder Executivo, Brasília, DF, 08 set. 2009

ORGANIZAÇÃO MUNDIAL DA SAÚDE. The rational use of drugs: report of the conference of experts. Nairobi 1985 Jul 25-29. Geneva: WHO; 1987

RANG, H.P. et al. Farmacologia. 5 $5^{\text {a }}$ Edição. Rio de Janeiro: Elsevier, (2004),904 p. ISBN - 853521368-6.

RODRIGUES M.A.P, FACCHINI L.A, LIMA M.S. Modificações nos padrões de consumo de psicofármacos em localidade do sul do Brasil. Rev Saude Pública. 2006;40(1):107-14.

TAMBLYN, R; LAPRISE, R.; HANLEY, J.A.; ABRAHAMOWICZ, M.; SCOTT, S.; MAYO, N.; HURLEY, J.; GRAD, R.; LATIMER, E.; PERREAULT, R.; MCLEOD, P.; HUANG, A.; LAROCHELLE P.; MALLET, L. Adverse events associated with prescription drug cost-sharing among poor and elderly persons. JAMA, v. 285, n. 4, p.421-9, 2001. 